\title{
PERFIL CLÍNICO Y EPIDEMIOLÓGICO DE PACIENTES CON ENFERMEDAD PULMONAR OBSTRUCTIVA CRÓNICA HOSPITALIZADOS EN UN CENTRO DE ALTA COMPLEIDAD DE LA CIUDAD DE MEDELLÍN, COLOMBIA, DURANTE EL AÑO 2015
}

Camilo Andrés Agudelo Vélez ${ }^{1}$, Lina María Martínez Sánchez ${ }^{2}$, Isabel Cristina Ortiz Trujillo ${ }^{3}$, María de los Ángeles Rodríguez Gázquez ${ }^{4}$, Mónica Zuluaga Quintero ${ }^{5}$, Natalia Perilla Hernández ${ }^{6}$, Felipe Hernández Restrepo ${ }^{6}$, Dayana Andrea Quintero Moreno $^{6}$, Camilo Ruiz Mejía ${ }^{6}$, María Alejandra Aristizábal Giraldo ${ }^{6}$

\section{Resumen}

Introducción. La enfermedad pulmonar obstructiva crónica (EPOC) es una condición que cursa con limitación del flujo aéreo espiratorio e inflamación crónica de las vías aéreas, y que representa un problema de salud pública a nivel mundial. Objetivo. Determinar el perfil clínico y epidemiológico de pacientes con EPOC en una institución hospitalaria de la ciudad de Medellín, Colombia. Metodología. se realizó un estudio transversal, con una muestra de 50 pacientes, con diagnóstico clínico o espirométrico de enfermedad pulmonar obstructiva crónica, atendidos de forma intrahospitalaria en una institución privada en Medellín durante el año 2015. A las variables cuantitativas se les calculó el promedio, desviación estándar y valores mínimo y máximo. A las cualitativas, medidas de nivel nominal y ordinal y se les estimaron proporciones. Resultados. La edad promedio fue de 73,5 $\pm 9,3$ años, el 52\% fueron mujeres. El promedio de tiempo de diagnóstico fue de 7,8 $\pm 1,3$ años. Las características clínicas más frecuentes fueron las siguientes: el 36\% tenía como clasificación estadio D para la enfermedad, el 34\% tenía VEF1 $<30 \%$, el $88 \%$ tenían antecedente de tabaquismo y el 52\% utilizaba oxígeno en casa. Conclusiones. La mayoría de nuestra población fue clasificada como GOLD categoría $\mathrm{D}$, con una limitación grave del flujo aéreo espiratorio ( $\mathrm{VEF} 1<30 \%$ ) y requerimiento de uso de oxígeno domiciliario. Lo anterior indica un inadecuado control de la enfermedad, debido, probablemente, al contexto intrahospitalario de los pacientes incluidos en el estudio.

${ }^{1}$ Médico, M.Sc. en Administración en Salud. Residente de Psiquiatría, Facultad de Medicina, Universidad Pontificia Bolivariana, Medellín, Colombia.

${ }^{2}$ Bacterióloga, Esp Hematología, M.Sc. en E. Docente titular y miembro del Grupo de Investigación en Medicina Interna, Facultad de Medicina, Universidad Pontificia Bolivariana, Medellín, Colombia.

${ }^{3}$ Bióloga, Ph.D. en Biología, M.Sc. en Biología. Docente titular y miembro del Grupo de Investigación en Biología de sistemas, Facultad de Medicina, Universidad Pontificia Bolivariana, Medellín, Colombia. ${ }^{4}$ Enfermera, Epidemióloga, Ph.D. en Salud Pública. Universidad de Antioquia, Medellín, Colombia.

${ }^{5}$ Médico, Esp. en Medicina Interna. Docente, Universidad Pontificia Bolivariana, Medellín, Colombia. ${ }^{6}$ Estudiantes de pregrado de Medicina, Universidad Pontificia Bolivariana, Medellín, Colombia. Grupo de Investigación Biología de Sistemas, Escuela de Ciencias de la Salud, Facultad de Medicina. 


\title{
CLINICAL AND EPIDEMIOLOGICAL PROFILE OF PATIENTS WITH CHRONIC OBSTRUCTIVE PULMONARY DISEASE HOSPITALIZED IN A HIGH COMPLEXITY CENTER OF THE CITY OF MEDELLÍN, COLOMBIA, IN 2015
}

\author{
Camilo Andrés Agudelo Vélez ${ }^{1}$, Lina María Martínez Sánchez ${ }^{2}$, Isabel Cristina \\ Ortiz Trujillo ${ }^{3}$, María de los Ángeles Rodríguez Gázquez ${ }^{4}$, Mónica Zuluaga Quin- \\ tero ${ }^{5}$, Natalia Perilla Hernández ${ }^{6}$, Felipe Hernández Restrepo ${ }^{6}$, Dayana Andrea \\ Quintero Moreno ${ }^{6}$, Camilo Ruiz Mejía ${ }^{6}$, María Alejandra Aristizábal Giraldo ${ }^{6}$
}

\section{Abstract}

Introduction. Chronic Obstructive Pulmonary Disease (COPD) is a condition that limits the air flow and produce chronic inflammation of the airways, which represents a public health problem worldwide. Objective. To determine the clinical and epidemiological profile of patients with COPD in a hospital of the city of Medellin, Colombia. Methodology. A cross-sectional study was carried out, with a sample of 50 subjects, who had a clinical or spirometric diagnosis of Chronic Obstructive Pulmonary Disease, receiving Inpatient care in a private institution in Medellin in 2015. It was calculated on quantitative variables, the average, standard deviation and minimum and maximum values. It was estimated on qualitative variables, measures of nominal and ordinal level and proportions. Results. The average age was 73.5 ) 9,3 years, $52 \%$ were women. The average of Diagnostic time was 7.8 ) 1,3 years. The most common clinical characteristics were the following: $36 \%$ had a stage D classification for the disease, $34 \%$ had FEV1 $<30 \%, 88 \%$ had a smoking history and $52 \%$ used oxygen at home. Conclusions. The majority of our population was classified as GOLD category D, with a severe limitation to breath (FEV1 <30\%) and had to use oxygen at home. The foregoing indicates that there is an inadequate control of the disease, due to the inpatient environment of the subjects involved in the study. 


\section{PERFIL CLÍNICO E EPIDEMIOLÓGICO DOS PACIENTES COM DOENÇA PULMONAR OBSTRUTIVA CRÔNICA (DPOC) HOSPITALIZADOS EM UM CENTRO DE ALTA COMPLEXIDADE DA CIDADE DE MEDELLÍN, COLÔMBIA, DURANTE 0 ANO 2015}

Camilo Andrés Agudelo Vélez ${ }^{1}$, Lina María Martínez Sánchez ${ }^{2}$, Isabel Cristina Ortiz Trujillo ${ }^{3}$, María de los Ángeles Rodríguez Gázquez ${ }^{4}$, Mónica Zuluaga Quintero ${ }^{5}$, Natalia Perilla Hernández ${ }^{6}$, Felipe Hernández Restrepo ${ }^{6}$, Dayana Andrea Quintero Moreno ${ }^{6}$, Camilo Ruiz Mejía ${ }^{6}$, María Alejandra Aristizábal Giraldo ${ }^{6}$

\section{Resumo}

Introdução. A doença pulmonar obstrutiva crônica (DPOC) é uma condição caracterizada por fluxo respiratório limitado e inflamação crônica das vias aéreas, e representa um problema de saúde pública em todo o mundo. Objetivo. Determinar o perfil clínico e epidemiológico dos pacientes com DPOC em uma instituição hospitalar da cidade de Medellín, Colômbia. Metodologia. Foi realizado um estudo transversal, com uma amostra de 50 pacientes, com diagnóstico clínico e espirométrico da doença pulmonar obstrutiva crônica, que receberam atenção hospitalar em uma instituição privada em Medellín durante o ano de 2015. Para as variáveis quantitativos foram calculados a média, desvio padrão e valores mínimo e máximo. Para medidas qualitativas de nível nominal e ordinal se estimaram proporções. Resultados. A idade média foi de 73,5 $\pm 9,3$ anos, 52\% eram mulheres. A média do tempo de diagnóstico foi de 7,8 $\pm 1,3$ anos. As características clínicas mais frequentes foram: $36 \%$ tinham classificação no estádio D para a doença, 34\% tinham VEF1 $<30 \%$, $88 \%$ tinham história de tabagismo e $52 \%$ usavam oxigênio em casa. Conclusões. A maioria da nossa população foi classificada como GOLD categoria $\mathrm{D}$, com uma limitação severa do fluxo de ar (VEF1 <30\%) e exigência de uso de oxigênio domiciliar. O que precede indica um controle inadequado da doença, devido, provavelmente, ao contexto hospitalar dos pacientes incluídos no estudo. 


\section{Introducción}

La enfermedad pulmonar obstructiva crónica (EPOC) es una condición que cursa con una limitación progresiva del flujo aéreo espiratorio e inflamación crónica a nivel pulmonar y que conlleva la aparición de síntomas persistentes, como disnea progresiva, que empeora con la actividad física y mejora con el reposo, expectoración y tos crónica con sibilancias recurrentes (1). Estas manifestaciones son consecuencia de cambios en las vías respiratorias, secundarios a una exposición prolongada a partículas o gases nocivos como los provenientes de combustibles de biomasa, contaminación atmosférica, y del humo de tabaco, el cual es el principal factor de riesgo (2).

Según la Organización Mundial de la Salud (OMS), alrededor de 65 millones de personas en el mundo padecen de EPOC, tanto en formas leves como graves. Tiene una prevalencia aproximada del $10,1 \%$ en individuos mayores de 40 años, es más común en hombres que en mujeres y se asocia a altas tasas de mortalidad, considerándose la tercera causa de muerte en el mundo $(3,4)$. El estudio PREPOCOL, llevado a cabo en 2007 en 5 ciudades de Colombia, determinó que, a nivel nacional, 9 de cada 100 personas mayores de 40 años tenían EPOC, representando una problemática de salud pública en nuestro medio (5).

El diagnóstico de EPOC se confirma a través de una curva flujo/volumen, con una relación VEF1/CVF (VEF1, volumen espiratorio forzado en 1 segundo $y$ CVF, capacidad vital forzada) posbron- codilatador menor de 0,70 , sumado a síntomas característicos de la enfermedad y factores de riesgo por exposición (2). La gravedad de la limitación del flujo aéreo se clasifica según el VEF1 en leve, moderada, grave y muy grave. No obstante, no se establece una relación proporcional con los síntomas; por esta razón, se utilizan diferentes escalas para la valoración del estado funcional y la clínica, tales como el cuestionario mMRC (escalada modificada de disnea), CAT (COPD Assessment Test) y el CCQ (COPD Control Questionnarie) (6).

La enfermedad se categoriza en 4 estadios (A, B, C o D), de acuerdo con el valor del VEF1, los síntomas y las exacerbaciones, definidas como episodios de inestabilidad que favorecen la progresión de la enfermedad. Así se determina el tratamiento y pronóstico de la enfermedad, siendo los pacientes estadio D quienes presentan mayor limitación del flujo aéreo, sintomatología y exacerbaciones $(2,7,8)$.

La información disponible respecto a las características clínicas y demográficas de la población colombiana con esta condición es limitada, por lo que el objetivo principal de este estudio fue determinar las características clínicas y epidemiológicas de pacientes con EPOC en una institución hospitalaria de la ciudad de Medellín, Colombia.

\section{Metodología}

Se realizó un estudio transversal, cuya muestra a conveniencia fueron 50 pacientes. Los criterios de inclusión fueron: pacientes mayores de 18 años, con 
diagnóstico clínico y/o espirométrico de EPOC que estaban hospitalizados en el servicio de medicina interna de una institución privada en Medellín a criterio del médico tratante, en el periodo de enero a diciembre del año 2015. El diagnóstico clínico se asigna a todo paciente con disnea, tos crónica o producción de esputo y/o antecedentes de exposición a factores de riesgo de la enfermedad; el espirométrico se obtiene a partir de la relación VEF1/CVF posbroncodilatador menor de 0,70 . Se excluyeron los pacientes con antecedentes personales de: cáncer de pulmón u otra enfermedad respiratoria crónica, trabajo en minas o trabajo con compuestos químicos inhalables. De igual forma, se excluyeron los pacientes cuyas historias clínicas no contaban con información suficiente para ser incluidas en el estudio y a aquellos que no deseaban participar en él.

La fuente de información fueron los pacientes, así como las historias clínicas que cumplieran con los criterios mencionados. Se realizó el registro en un formulario, diseñado previamente por los investigadores. El análisis de la información se llevó a cabo en el programa SPSS vr. 20. El promedio de las variables cuantitativas se calculó con su respectiva desviación estándar y valores mínimo y máximo. Se estimaron proporciones para las variables cualitativas.
La investigación tuvo el aval del comité de ética de la institución donde se llevó a cabo. Mediante dicha autorización fue posible revisar la información contenida en la historia clínica. Se solicitó consentimiento informado a los pacientes incluidos en la investigación.

\section{Resultados}

Participaron 50 pacientes con EPOC, en los que predominaron las siguientes características sociodemográficas: edad promedio de 73,5 $\pm 9,3$ años (mínimo 57 y máximo 93), la mayoría eran de sexo femenino (52\%), el 50\% eran jubilados y el $80 \%$ residía en la ciudad de Medellín (Tabla 1).

En cuanto a las características clínicas, el promedio de tiempo desde el diagnóstico fue de 7,8 1 ,3 años, según la información de la historia clínica y el interrogatorio directo al paciente (míni$\mathrm{mo}=$ menos de un año y máximo $=50$ años) y el $100 \%$ de ellos fue tratado en el servicio de medicina interna de la institución. Las características clínicas más frecuentes fueron: clasificación de gravedad de la enfermedad EPOC D (36\%), VEF1 $<30 \%(34 \%)$, el $88 \%$ tenía antecedentes de tabaquismo, y el 52\% utilizaba oxígeno en casa. De igual forma, la tos y la disnea fueron los síntomas más frecuentes. Otras características clínicas se evidencian en la Tabla 1. 
INVESTIGACIONES ANDINA No. 37, Vol. 20

Tabla 1. Características clínicas de 50 pacientes con EPOC

\begin{tabular}{|c|c|}
\hline Variable & $n(\%)$ \\
\hline Servicio tratante: medicina interna & $50(100)$ \\
\hline \multicolumn{2}{|l|}{ Diagnóstico de EPOC } \\
\hline $\begin{array}{l}\text { Confirmado por espirometría (registro } \\
\text { antiguo reportado en la historia clínica) }\end{array}$ & $43(86)$ \\
\hline Sin dato espirométrico & $7(14)$ \\
\hline \multicolumn{2}{|l|}{ Clasificación del EPOC } \\
\hline GOLD A & $2(4)$ \\
\hline GOLD B & $6(12)$ \\
\hline GOLDC & $13(26)$ \\
\hline GOLD D & $18(36)$ \\
\hline Sin dato & $11(22)$ \\
\hline \multicolumn{2}{|l|}{ Espirometría } \\
\hline GOLD 1 (VEF1 $\geq 80 \%)$ & $3(6)$ \\
\hline GOLD 2 (VEF1 50\%-79\%) & $6(12)$ \\
\hline GOLD 3 (VEF1 30\%-49\%) & $12(24)$ \\
\hline GOLD 4 (VEF1 <30\%) & $17(34)$ \\
\hline Sin dato & $12(24)$ \\
\hline \multicolumn{2}{|l|}{ Tabaquismo } \\
\hline Sí & $44(88)$ \\
\hline \multicolumn{2}{|l|}{$\begin{array}{l}\text { Uso de oxígeno domiciliario (mínimo } 12 \\
\text { horas/día) }\end{array}$} \\
\hline Sí & $26(52)$ \\
\hline No & $24(48)$ \\
\hline
\end{tabular}


Continuación Tabla 1. Características clínicas de 50 pacientes con EPOC

\begin{tabular}{|c|c|}
\hline Variable & $n(\%)$ \\
\hline \multicolumn{2}{|l|}{ Síntomas } \\
\hline Tos $^{*}$ & $47(94)$ \\
\hline Expectoración** & $40(80)$ \\
\hline Disnea*** & $44(88)$ \\
\hline \multicolumn{2}{|l|}{ Signos } \\
\hline Prolongación de fase espiratoria & $41(82)$ \\
\hline Disminución de ruidos respiratorios" & $47(94)$ \\
\hline Tórax en tonel" " & $16(32)$ \\
\hline Uso músculos accesorios" "॥" & $4(8)$ \\
\hline Crépitos $\Delta$ y sibilancias $\Delta \Delta$ & $18(36)$ \\
\hline Antecedente de hospitalización $\Delta \Delta \triangle$ & $50(100)$ \\
\hline
\end{tabular}

*Tos: expulsión brusca, violenta y ruidosa del aire contenido en los pulmones producida por la irritación de las vías respiratorias, puede ser intermitente $y$ no productiva.

**Expectoración: expulsión de flemas u otras secreciones formadas en las vías respiratorias, mediante la tos. Cualquier patrón de producción de esputo puede indicar EPOC.

***Disnea: dificultad respiratoria que debe ser persistente, progresiva a lo largo del tiempo y que empeora con el ejercicio. "Disminución de los ruidos respiratorios: disminución del murmullo vesicular por reducción del flujo aéreo.

“ "Tórax en tonel: tórax redondeado y abultado en el que predomina un au- mento del diámetro antero posterior de la caja torácica.

“ " "Uso de músculos accesorios: uso del esternocleidomastoideo, intercostales y escalenos en la respiración

$\Delta$ Crépitos: es un ruido crujiente, similar al que se produce cuando se despega un velcro, puede estar presente en ambas fases de la respiración.

$\Delta \Delta$ Sibilancias: se generan por estenosis de vías aéreas de pequeño calibre. Los ruidos son como chillidos agudos o silbidos que se producen tanto en inspiración como espiración.

$\Delta \Delta \Delta$ Antecedente de hospitalización: cualquier hospitalización previa por exacerbación del EPOC en cualquier momento de la vida. 


\section{Discusión}

Describimos una mayor prevalencia de la EPOC en pacientes de sexo femenino y de mayor edad a lo reportado en otras cohortes. Liu et al. (9) reportan una prevalencia de EPOC en el sexo femenino de un 53,6\%, similar a la nuestra, que fue de $52 \%$. Es posible que dicho resultado se relacione con una mayor susceptibilidad de las mujeres a los efectos nocivos del tabaco (9). La edad promedio de presentación de la enfermedad descrita por Bozek et al. (10) fue de $66,5 \pm 5,9$, similar a la reportada por Mitsiki et al., en el estudio GOLDEN (68 años, RIC 60,0-75,0), y ambas son menores a la reportada en nuestro estudio de 73,5 $\pm 9,3$ años (11).

Probablemente, esto se explica por el tipo de pacientes incluidos, quienes fueron atendidos de forma intrahospitalaria y generalmente tienen mayor comorbilidad en relación con la edad y al número de exacerbaciones. La mayoría de los individuos tenían antecedentes de tabaquismo (88\%), lo cual concuerda con lo reportado por otros autores como Blasi et al. (91,7\%) (12) y Morales- Menéndez et al. (61,4\%), así que se reafirma la importancia de la exposición al humo de tabaco como principal factor de riesgo, a pesar de no ser el único. Respecto a las manifestaciones clínicas, la tos y disnea son el síntoma más prevalente, al igual que lo descrito en literatura mundial, siendo la espiración prolongada y la disminución de los ruidos respiratorios los signos más
La mayoría de nuestra población (86\%) tuvo confirmación espirométrica del diagnóstico de EPOC, mientras que un $14 \%$ no tenía información disponible acerca de esta prueba. Por consiguiente, no descartamos que otros diagnósticos diferenciales fueran omitidos y pudieran ser un factor que causara confusión en el momento de clasificar el estadio de la enfermedad, gravedad de los síntomas y pronóstico. Sin embargo, es importante resaltar que, aun sin tener espirometría, los factores de riesgo y la clínica pueden orientar en el seguimiento clínico. Asimismo, con la escala de disnea y exacerbaciones es posible realizar ajustes en la terapia (13).

Sobre la clasificación de la enfermedad, Li-Cher et al. (14), realizaron un estudio en 120 pacientes hospitalizados por exacerbación de la EPOC en el que reportaron una mayor prevalencia de categoría B $(63,3 \%)$, contrario a lo reportado en nuestro estudio, donde la mayoría se clasificaron en categoría D (36\%). Ambos estadios se esperan de pacientes hospitalizados, dado que tienen mayor número de exacerbaciones con pobre control de la enfermedad. No obstante, llama la atención que, en nuestro medio, el estadio de mayor frecuencia es el D; esto es muy probablemente dado por las condiciones de salud de la población y por las situaciones adyacentes a la misma enfermedad como, por ejemplo, la adherencia a la terapia inhalada, la garantía en la entrega de medicamentos controladores de la enfermedad y los cambios en estilo de vida y hábitos, entre ellos, la suspensión del tabaquismo. Estos factores influyen en la progresión de la EPOC y el control 
de los síntomas, así como el impacto en exacerbaciones.

De igual forma, una proporción significativa de nuestra población no fue clasificada (22\%), en gran parte, por la ausencia de resultados de pruebas espirométricas, razón que también puede influir en este hallazgo. Sin embargo, el 35,4\% de los pacientes que tenían el reporte de curva flujo volumen tenía un VEF $1<30 \%$, dato que difiere del reportado por Mitsiki et al (11): (VEF1 $50 \%-79 \%$ ) en un $35,4 \%$ de la población, y por Blasi et al. (12), donde el $18 \%$ los pacientes fueron clasificados como GOLD. Adicionalmente, la mayor proporción de nuestra población (52\%) utilizaba oxígeno domiciliario, contrario a lo reportado en el estudio GOLDEN por Mitsiki et al. (11), donde solo un $17,1 \%$ requería oxígeno en casa, lo que demuestra que, además de los factores socioeconómicos y culturales que

\section{Referencias}

1. Pavod I., et al. Mepolizumab for Eosinophilic Chronic Obstructive Pulmonary Disease. N Engl J Med. 2017;377(17):1613-29.

2. Roversi S., Corbetta L., Clini E. GOLD 2017 recommendations for COPD patients: toward a more personalized approach. COPD Research and Practice. 2017;3(5):1-6.

3. Vogelsinger $H_{\text {., et al. Efficacy and safe- }}$ ty of nasal high-flow oxygen in COPD patients. BMC Pulm Med. 2017;17(143):1-8. puedan estar relacionados, los fenotipos de EPOC en nuestro medio tienen una enfermedad de mayor gravedad.

\section{Conclusiones}

Las características demográficas y clínicas de nuestros pacientes son muy similares a lo descrito en la literatura, si bien la mayor frecuencia del estadio D y el requerimiento de uso de oxígeno domiciliario demuestran una presentación más grave de la enfermedad en el medio. Es posible que esto se relacione no sólo con el fenotipo sino también con factores sociales, económicos $y$ culturales que interactúan con el proceso de la enfermedad y que son objeto de intervención por parte del médico y el sistema de salud para garantizar una mayor adherencia al tratamiento, un mejor seguimiento y un mejor impacto en el pronóstico de estos pacientes.

4. Zhou Y., et al. Tiotropium in Early-Stage Chronic Obstructive Pulmonary Disease. N Engl J Med. 2017;377(10):923-35.

5. Pineda S., Ramos V., Cadavid D. Calidad de vida en pacientes con Enfermedad Pulmonar Obstructiva. Universidad y Salud. 2016;18(3):482-93.

6. Huang WC., et al. Features of COPD patients by comparing CAT with mMRC: a retrospective, cross-sectional study. NPJ Prim Care Respir Med. 2015;25:15063. 
7. Calle M., Morales B. y Rodríguez J. Exacerbación de la EPOC. Arch Bronconeumol. 2010;46(7):21-25.

8. Leung J., Sin D. Asthma-COPD overlap syndrome: pathogenesis, clinical features, and therapeutic targets. BMJ [Internet]. 2017 [Citado año mes día];358:1-14. Disponible en: https://www.ncbi.nlm.nih.gov/ pubmed/28947632

9. Liu Y., et al. Smoking duration, respiratory symptoms, and COPD in adults aged $\geq 45$ years with a smoking history. Int J Chron Obstruct Pulmon Dis. 2015;10(1):1409-16.

10. Bozek A., Rogala B., Bednarski P. Asthma, COPD and comorbidities in elderly people. J Asthma. 2016;53(9):943-7.

11. Mitsiki E., et al. Characteristics of prevalent and new COPD cases in Greece: the GOLDEN study. Int J Chron Obstruct Pulmon Dis. 2015;10(1):1371-82.

12. Blasi F., et al. Clinical Characterization and Treatment Patterns for the Frequent Exacerbator Phenotype in Chronic Obstructive Pulmonary Disease with Severe or Very Severe Airflow Limitation. COPD. 2017;14(1):15-22.

13. Sinha B., Vibha, Singla R., Chowdhury R. An epidemiological profile of chronic obstructive pulmonary disease: a community-based study in Delhi. J Postgrad Med. 2017;63(1):29-35.

14. Li-Cher L., Choo-Khoon O. Detailed characterization of hospitalized COPD patients in relation to combined COPD assessment by GOLD. Chronic Obstructive Pulmonary Diseases [Internet]. 2016 [Citado año mes día];1(4):1-9. 